\title{
TEACHING PROGRAMME IS EFFECTIVE IN INCREASING KNOWLEDGE TO PREVENT MALNUTRITION IN CHILDREN
}

\section{Neha Rehalia}

\section{Vivek Chaudhary*}

\author{
Pediatrician, Civil hospital Shahpur, Kangra (HP).
}

Anaesthesiologist, Zonal hospital Dharamshala, Kangra (HP). ${ }^{*}$ Corresponding Author

ABSTRACT Introduction: In developing countries, malnutrition among children is a major public-health issue. It is one of the most serious global risk factor for illness and death. Malnutrition during children has an impact later in life as it is associated with significant functional impairment, reduced work capacity and decreased economic productivity. Malnourished children are more likely to suffer from delayed psychological development, poor school performance and lower intellectual achievements.

Aim: Effectiveness of Teaching Programme on Malnutrition and its Prevention in under-five children.

Methods: Fifty children for the were enrolled through simple randomization method in this study.

Results: After administration of teaching program, 92\% subjects had adequate knowledge and $8 \%$ had moderate knowledge. None of the subjects had inadequate level of knowledge showing effectiveness of teaching program. Post-test overall knowledge score was significantly higher in comparison to pre-test overall knowledge score (32.20 \pm 8.77 vs. $8.14 \pm 10.99$; $\mathrm{P}=0.01$ ). Age, gender, nationality, family income, fathers' occupation, mothers' education, religion, students' status, previous knowledge, and source of information were significantly associated with the post-test level of knowledge on malnutrition and its prevention.

Conclusion: Teaching programme is effective in increasing knowledge of adolescent regarding malnutrition and its prevention in under-five children.

\section{KEYWORDS :}

\section{INTRODUCTION}

Malnutrition in preschool children is a significant problem and has been identified by the World Health Organization (WHO) as the most lethal form of disease which indirectly causes an annual death of at least 5 million children worldwide. ${ }^{1}$ Malnutrition is widely recognized as a major health problem in developing countries. It is wide spread in rural, tribal and urban slum areas. Growing children are most vulnerable to its consequences. Anthropometry is a simple field technique for evaluating physical growth and nutritional status of the children.

The United Nations Food and Agriculture Organization (FAO), estimates that nearly 870 million people of the 7.1 billion people in the world, or one in eight, were suffering from chronic undernourishment in 2010-2012. Almost all the hungry people, 852 million, live in developing countries, representing 15 percent of the population of developing counties.

At present $2 / 3^{\text {rd }}$ of the deaths of the children around the world are directly or indirectly associated with nutritional in deficiencies. Nearly half of India's children- approximately 60 million - are underweight, $45 \%$ have stunted growth (too short for their age), $20 \%$ are wasted (too thin for their height, indicating acute malnutrition), $75 \%$ are anaemic, and $57 \%$ are deficient in Vitamin A.

The findings of the third National Family Health Survey (NFHS-3) revealed an unacceptable prevalence of malnutrition in our children: $42.5 \%$ of our children under the age of five years are underweight (low weight for age), $48 \%$ of our children are stunted (low height for age - chronically malnourished), $19.8 \%$ of our children are wasted (low weight for height - acutely malnourished) and in poorer states the situation is even worse with over $50 \%$ of children underweight. ${ }^{4}$

Malnutrition is both a cause and consequence of disease and illness and there can be many contributing factors. Whilst some causes of malnutrition might be the result of underlying ill health, disease or the body's inability to absorb nutrients, malnutrition can also be linked to other experiences or factors in a person's life.
The costs of malnutrition runs into billions of pounds in spite of proven interventions that can prevent identify and manage the problem and risks promptly and thereby reduce the human suffering and the astronomical associated costs.

Studies have revealed that severe degree of malnutrition can be reduced by practice of exclusive breast feeding, introduction of timely complementary feeding, education for maintaining personal hygiene, proper implementation of UIP immunization, periodic de-worming, standard case management of diarrhea and ARI as well as continuation of feeding during illness among under-five children.

With reference to the above studies malnutrition is quite prevalent in our state and the major cause is lack of knowledge among caretakers which needs to be addressed. Hence, the study was aimed to evaluate effectiveness of Teaching Programme (CATP) on malnutrition and its prevention in under five children.

\section{Subjects And Methods}

Fifty children were enrolled through simple randomization method in this study. The students were included in the study if ready for consent to participate in the study.

\section{Data Collection Tool}

A structured knowledge questionnaire was developed through extensive study of literature and discussion with experts. Content validity of the tool was confirmed through nine experts. The tool consisted of two sections, and had a total of 36 questions.

Section I contained questions related to demographic variables of the study population. Demographic variables include age, sex, religion, income of family (father), educational status of parents (father and mother), occupation of parents (father and mother), student's status, previous knowledge and its source, and source of information.

Section II contained items related to knowledge regarding malnutrition and its prevention.

Scoring Procedure

For knowledge items, each correct answer was given a score of 
'one' and incorrect answer a score of 'zero'. The knowledge questionnaire has 36 item regarding Malnutrition and its Prevention for the correct answer given score of "one" So, the total score was 36 for the knowledge questionnaire.

\section{Statistical Analysis}

Data were presented as frequency, percentage, mean, and standard deviation. Categorical variables were compared using Chi square test. Paired t-test was used to compare pretest and post-test knowledge score. $\mathrm{P}$ value $<0.05$ was considered significant. Statistical analysis was performed using SPSS v21.

\section{RESULTS}

\section{General Characteristics}

Table 1 shows general characteristics according to age, sex, family income of parents, occupation of parents, educational status of parents, religion, student's place of residence, and previous knowledge on malnutrition and source of information of the subjects. Among 50 subjects, majority $52.0 \%$ of subjects belong to 16 years, $48.0 \%$ belongs to 17 years. Majority $66 \%$ of subjects were male subjects and $34 \%$ were female subjects. $98 \%$ of subjects were Indian.

Table 1. Demographic Characteristics

\begin{tabular}{|c|c|c|c|}
\hline \multirow[t]{2}{*}{ Characteristics } & \multirow[t]{2}{*}{ Category } & \multicolumn{2}{|l|}{ Subjects } \\
\hline & & Frequency & Percent \\
\hline \multirow[t]{2}{*}{ Age (in years) } & 16 & 26 & 52 \\
\hline & 17 & 24 & 48 \\
\hline \multirow[t]{2}{*}{ Gender } & Male & 17 & 34 \\
\hline & Female & 27 & 90 \\
\hline \multirow[t]{2}{*}{ Nationality } & Indian & 49 & 98 \\
\hline & Foreigner & 1 & 2.0 \\
\hline \multirow{7}{*}{$\begin{array}{l}\text { Family income } \\
\text { (KSES) father } \\
\text { (in rupees) }\end{array}$} & $>32,050$ & 12 & 24 \\
\hline & $16,020-32,049$ & 12 & 24 \\
\hline & $12,020-16,019$ & 12 & 24 \\
\hline & $8,010-12,019$ & 9 & 18 \\
\hline & $4,810-8,009$ & 2 & 4 \\
\hline & $1,601-4,809$ & 2 & 4 \\
\hline & $<1,600$ & 1 & 2 \\
\hline \multirow{7}{*}{$\begin{array}{l}\text { Occupation of } \\
\text { Family (KSES) } \\
\text { Father }\end{array}$} & Profession & 20 & 40 \\
\hline & Semi- Profession & 5 & 10 \\
\hline & Clerical, shop-owner & 15 & 30 \\
\hline & Skilled worker & 5 & 10 \\
\hline & Semi-skilled worker & 0 & 0 \\
\hline & Unskilled worker & 2 & 4 \\
\hline & Unemployed & 3 & 6 \\
\hline \multirow{7}{*}{$\begin{array}{l}\text { Occupation of } \\
\text { Family (KSES) } \\
\text { Mother }\end{array}$} & Profession & 6 & 12 \\
\hline & Semi- Profession & 4 & 8 \\
\hline & Clerical, shop-owner & 10 & 20 \\
\hline & \begin{tabular}{|l|} 
Skilled worker \\
\end{tabular} & 11 & 22 \\
\hline & Semi-skilled worker & 0 & 0 \\
\hline & Unskilled worker & 0 & 0 \\
\hline & Unemployed & 19 & 38 \\
\hline \multirow{7}{*}{$\begin{array}{l}\text { Education } \\
\text { status(KSES) } \\
\text { Father }\end{array}$} & Profession & 7 & 14 \\
\hline & $\begin{array}{l}\text { Graduate or post } \\
\text { graduate }\end{array}$ & 12 & 24 \\
\hline & $\begin{array}{l}\text { Intermediate or post } \\
\text { high school }\end{array}$ & 9 & 18 \\
\hline & \begin{tabular}{|l|} 
High school \\
certificate
\end{tabular} & 18 & 26 \\
\hline & \begin{tabular}{|l|}
$\begin{array}{l}\text { Middle school } \\
\text { certificate }\end{array}$ \\
\end{tabular} & 2 & 4 \\
\hline & \begin{tabular}{|l|} 
Primary school \\
certificate
\end{tabular} & 1 & 2 \\
\hline & Illiterate & 1 & 2 \\
\hline \multirow{2}{*}{$\begin{array}{l}\text { Education } \\
\text { status (KSES) } \\
\text { mother }\end{array}$} & Profession & 2 & 4 \\
\hline & $\begin{array}{l}\text { Graduate or post } \\
\text { graduate }\end{array}$ & 16 & 32 \\
\hline
\end{tabular}

\begin{tabular}{|c|c|c|c|}
\hline & $\begin{array}{l}\text { Intermediate or post } \\
\text { high school }\end{array}$ & 7 & 14 \\
\hline & $\begin{array}{l}\text { High school } \\
\text { certificate }\end{array}$ & 17 & 34 \\
\hline & $\begin{array}{l}\text { Middle school } \\
\text { certificate }\end{array}$ & 2 & 4 \\
\hline & $\begin{array}{l}\text { Primary school } \\
\text { certificate }\end{array}$ & 2 & 4 \\
\hline & Illiterate & 4 & 8 \\
\hline \multirow[t]{4}{*}{ Religion } & Hinduism & 50 & 100 \\
\hline & \begin{tabular}{|l|} 
Islam \\
\end{tabular} & 0 & 0 \\
\hline & Christianity & 0 & 0 \\
\hline & Others & 0 & 100 \\
\hline \multirow{3}{*}{$\begin{array}{l}\text { Students place } \\
\text { of Residence }\end{array}$} & Day Scholar & 45 & 90 \\
\hline & Paying guest & 2 & 4 \\
\hline & Hosteller & 3 & 6 \\
\hline \multirow{2}{*}{\begin{tabular}{|l|} 
Previous \\
Knowledge \\
\end{tabular}} & Yes & 13 & 26 \\
\hline & No & 37 & 74 \\
\hline \multirow[t]{3}{*}{$\begin{array}{l}\text { Source of } \\
\text { information }\end{array}$} & $\begin{array}{l}\text { Magazines/Books/ } \\
\text { Posters }\end{array}$ & 16 & 32 \\
\hline & TV/Radio/Internet & 32 & 64 \\
\hline & $\begin{array}{l}\text { Friends/Neighbor/ } \\
\text { Health Personnel }\end{array}$ & 2 & 4 \\
\hline
\end{tabular}

$24.0 \%$ of subject's father had income Rs. $\geq 32050,24.0 \%$ had Rs. 16020 - 32049, $24.0 \%$ had Rs. $12020-16019$ and $18.0 \%$ had 8010 - 12019, 4\% had Rs. 4810 - 8009 income, 4\% had $1601-4809$ and $2 \%$ had Rs. $<1,600$ income respectively. $40 \%$ fathers were professional, $30.0 \%$ were as clerical, shop-owner farmer, $10 \%$ were semi-professions, $10 \%$ were skilled worker and $6 \%$ were unemployed and $4 \%$ were unskilled worker and none were semi skilled worker respectively. $38 \%$ mothers were unemployed, $22 \%$ were skilled worker, $20 \%$ clerical, shopowner farmer, $12 \%$ were professional, $8 \%$ semi- professional and none were semi- skilled worker and unskilled worker respectively.

$26.0 \%$ father had completed high school certificate, $24.0 \%$ had completed graduate or post graduate, $18.0 \%$ had completed Intermediate or post high school diploma, $14.0 \%$ were Profession, $4.0 \%$ had completed middle school certificate, $2.0 \%$ had completed primary school certificate and $2.0 \%$ were illiterate respectively. $34 \%$ mothers had completed high school certificate, $32 \%$ had completed graduate or post graduate, $14 \%$ had completed intermediate or post high school diploma, $8 \%$ were illiterate, and $4.0 \%$ had completed profession, middle school certificate and primary school certificates respectively.

All of the subjects were Hindu. 90\% were day scholar, $6.0 \%$ were hosteller and $4 \%$ were paying guest. $74 \%$ of the subjects were not exposed to knowledge on malnutrition and its prevention and $26 \%$ of the subjects were exposed to knowledge on malnutrition and its prevention. $64 \%$ of subjects had information from TV/Radio/Internet, $32 \%$ had information from magazines/books/posters and $4 \%$ of subjects had information from Friends/Neighbors/Health personnel.

\section{Comparison Of Knowledge Level}

We classified knowledge level on the basis of total score. Subjects who scored $\geq 65 \%$ were considered to have adequate knowledge. Scores with $41 \%$ to $65 \%$ were considered moderate knowledge and scoring $<40 \%$ was considered inadequate knowledge.

We observed that $90 \%$ of the subjects had inadequate knowledge in pre-test. While only $8 \%$ and $2 \%$ subjects had moderate and adequate level of knowledge respectively. After administration of teaching program, 92\% subjects had adequate knowledge and $8 \%$ had moderate knowledge. None of the subjects had inadequate level of knowledge showing effectiveness of teaching program (fig l). 


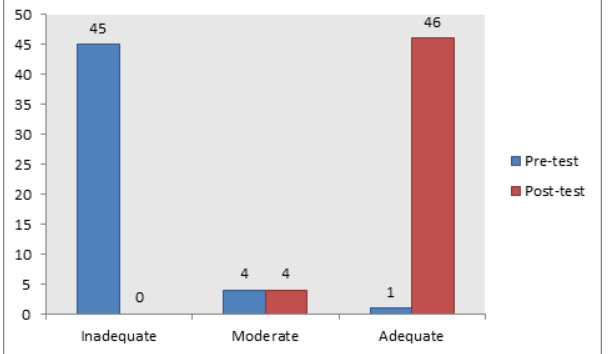

Fig 1. Distribution Of Subjects On The Basis Of Level Of Knowledge In Pre-test And Post-test; X-axis Shows Number Of Subjects. Y-axis Shows Level Of Knowledge In Pre- And Post-test

\section{Comparison Of Knowledge Score}

We also evaluated effectiveness of total knowledge score following administration of teaching program. We observed that post-test overall knowledge score was significantly higher in comparison to pre-test overall knowledge score $(32.20 \pm 8.77$ vs. $8.14 \pm 10.99 ; \mathrm{P}=0.01)$.

We also evaluated knowledge of individual aspects on knowledge scores related to malnutrition and its prevention.
We observed that all the aspects of the knowledge were significantly higher following administration of the teaching program (table 2).

Table 2: Comparison Of Individual Aspects Of Knowledge

\begin{tabular}{|l|l|l|l|l|l|}
\hline \multirow{2}{*}{ Aspect } & Pre-test & \multicolumn{2}{l|}{ Post-test } & P value \\
\cline { 2 - 5 } & Mean & SD & Mean & SD & \\
\hline $\begin{array}{l}\text { Introduction, definition } \\
\text { and incidence }\end{array}$ & 1.20 & \pm 1.78 & 5.20 & \pm 1.32 & $<0.05$ \\
\hline $\begin{array}{l}\text { Classification and } \\
\text { Causes }\end{array}$ & 2.53 & \pm 3.65 & 8.98 & \pm 2.72 & $<0.01$ \\
\hline $\begin{array}{l}\text { Diagnosis and sign } \\
\text { and symptoms }\end{array}$ & 1.43 & \pm 1.92 & 5.10 & \pm 1.75 & $<0.001$ \\
\hline $\begin{array}{l}\text { Management and } \\
\text { prevention }\end{array}$ & 2.98 & \pm 3.64 & 12.92 & \pm 2.98 & $<0.01$ \\
\hline
\end{tabular}

Association Of Demographic Variables With Knowledge Level

We also evaluated relation of the demographic variables with post-test knowledge level. We observed that age, gender nationality, family income, fathers' occupation, mothers education, religion, students' status, previous knowledge, and source of information were significantly associated with the post-test level of knowledge on malnutrition and its prevention $(\mathrm{P}<0.05)$ (table 3 ).

Table 20: Association Of Post-test Knowledge Level Of Subjects With Demographic Variable N=50

\begin{tabular}{|c|c|c|c|c|c|c|c|c|}
\hline \multirow{3}{*}{\multicolumn{2}{|c|}{ Aspect wise comparison }} & \multicolumn{6}{|c|}{ Knowledge Score } & \multirow[t]{3}{*}{ Pearson Chi-square test } \\
\hline & & \multicolumn{2}{|c|}{ Inadequate } & \multicolumn{2}{|c|}{ Moderate } & \multicolumn{2}{|c|}{ Adequate } & \\
\hline & & $\mathrm{n}$ & $\%$ & n & $\%$ & $\mathrm{n}$ & $\%$ & \\
\hline \multirow[t]{2}{*}{ Age in year } & 16 Yrs old & 0 & 0 & 2 & 50 & 24 & 51.17 & \multirow[t]{2}{*}{$\chi 2=2.52 \mathrm{P}=.01 \mathrm{Df} 2$, significant } \\
\hline & 17 Yrs ole & 0 & 0 & 2 & 50 & 22 & 47.82 & \\
\hline \multirow[t]{2}{*}{ Gender } & Male & 0 & 0 & 3 & 75 & 30 & 65.21 & \multirow[t]{2}{*}{$\chi 2=3.29 \mathrm{P}=0.02 \mathrm{Df} 2$, significant } \\
\hline & Female & 0 & 0 & 1 & 25 & 16 & 34.0 & \\
\hline \multirow[t]{2}{*}{ Nationality } & Indian & 0 & 0 & 4 & 100 & 45 & 97.82 & \multirow[t]{2}{*}{$\chi 2=1.03, P=0.0220 \mathrm{Df} 2$, significant } \\
\hline & Foreigner & 0 & 0 & 0 & 0 & 1 & 2.17 & \\
\hline \multirow[t]{7}{*}{ Family Income } & Rs. 32,050 & 0 & 0 & 1 & 20 & 11 & 23.9 & \multirow[t]{7}{*}{$\chi 2=6.43 \mathrm{P}=0.0290 \mathrm{Df} 6$, significant } \\
\hline & \begin{tabular}{|l|} 
Rs.16020-32039 \\
\end{tabular} & 0 & 0 & 1 & 20 & 11 & 23.9 & \\
\hline & Rs. 12020-16019 & 0 & 0 & 2 & 50 & 10 & 21.7 & \\
\hline & \begin{tabular}{|l|} 
Rs.8010-12019 \\
\end{tabular} & 0 & 0 & 0 & 0 & 9 & 19.5 & \\
\hline & Rs.4810-8009 & 0 & 0 & 0 & 0 & 2 & 4.3 & \\
\hline & Rs.1601-4809 & 0 & 0 & 0 & 0 & 2 & 4.3 & \\
\hline & R. $<1600$ & 0 & 0 & 0 & 0 & 1 & 2.1 & \\
\hline \multirow[t]{7}{*}{ Occupation of (Father) } & Profession & 0 & 0 & 2 & 50 & 18 & 39.13 & \multirow[t]{7}{*}{$\chi 2=3.32 \mathrm{P}=0.0390 \mathrm{Df} 6$, significant } \\
\hline & \begin{tabular}{|l|} 
Semi-Profession \\
\end{tabular} & 0 & 0 & 0 & 0 & 5 & 10.8 & \\
\hline & Clerical & 0 & 0 & 1 & 25 & 14 & 30.43 & \\
\hline & \begin{tabular}{|l} 
Skilled worker \\
\end{tabular} & 0 & 0 & 1 & 25 & 4 & 8.69 & \\
\hline & \begin{tabular}{|l|} 
Semi-Skilled \\
\end{tabular} & 0 & 0 & 0 & 0 & 0 & 0 & \\
\hline & \begin{tabular}{|l|} 
Unskilled \\
\end{tabular} & 0 & 0 & 0 & 0 & 2 & 4.3 & \\
\hline & Unemployed & 0 & 0 & 0 & 0 & 3 & 6.5 & \\
\hline \multirow[t]{7}{*}{ Occupation of (Mother) } & Profession & 0 & 0 & 0 & 0 & 6 & 13.04 & \multirow{7}{*}{$\begin{array}{l}\chi 2=6.47 \mathrm{P}=0.65 \mathrm{df} 6, \text { Non- } \\
\text { significant }\end{array}$} \\
\hline & Semi-Profession & 0 & 0 & 1 & 25 & 3 & 6.5 & \\
\hline & Clerical & 0 & 0 & 1 & 25 & 9 & 19.56 & \\
\hline & Skilled worker & 0 & 0 & 1 & 25 & 10 & 21.73 & \\
\hline & \begin{tabular}{|l} 
Semi-Skilled \\
\end{tabular} & 0 & 0 & 0 & 0 & 0 & 0 & \\
\hline & Unskilled & 0 & 0 & 0 & 0 & 0 & 0 & \\
\hline & Unemployed & 0 & 0 & 1 & 25 & 18 & 39.13 & \\
\hline \multirow[t]{7}{*}{ Education (Father ) } & Profession & 0 & 0 & 1 & 25 & 6 & 13.04 & \multirow{7}{*}{$\begin{array}{l}\chi 2=5.42 \mathrm{P}=0.719 \mathrm{df} 6, \text { Non } \\
\text { significant }\end{array}$} \\
\hline & Graduate & 0 & 0 & 1 & 25 & 11 & 23.91 & \\
\hline & \begin{tabular}{|l|} 
Intermediate \\
\end{tabular} & 0 & 0 & 0 & 0 & 9 & 19.56 & \\
\hline & High school & 0 & 0 & 2 & 50 & 17 & 36.95 & \\
\hline & Middle school & 0 & 0 & 0 & 0 & 2 & 4.3 & \\
\hline & Primary school & 0 & 0 & 0 & 0 & 1 & 2.1 & \\
\hline & Illiterate & 0 & 0 & 0 & 0 & 1 & 2.1 & \\
\hline \multirow[t]{6}{*}{ Education (Mother) } & Profession & 0 & 0 & 0 & 0 & 2 & 4.3 & \multirow[t]{6}{*}{$\chi 2=3.23 \mathrm{P}=0.0390 \mathrm{Df} 6$, significant } \\
\hline & \begin{tabular}{|l|} 
Graduate \\
\end{tabular} & 0 & 0 & 2 & 50 & 5 & 10.8 & \\
\hline & Intermediate & 0 & 0 & 1 & 25 & 6 & 13.04 & \\
\hline & High school & 0 & 0 & 1 & 25 & 16 & 34.78 & \\
\hline & Middle school & 0 & 0 & 0 & 0 & 2 & 4.3 & \\
\hline & Primary school & 0 & 0 & 0 & 0 & 2 & 4.3 & \\
\hline
\end{tabular}




\begin{tabular}{|c|c|c|c|c|c|c|c|c|}
\hline & Illiterate & 0 & 0 & 0 & 0 & 4 & 8.69 & \\
\hline Religion & Hinduism & 0 & 0 & 4 & 100 & 46 & 100 & $\chi 2=1.58 \mathrm{P}=0.01 \mathrm{Dfl}$, significant \\
\hline \multirow[t]{3}{*}{ Students status } & Day scholar & 0 & 0 & 3 & 75 & 42 & 91.30 & \multirow[t]{3}{*}{$\chi 2=2.28 \mathrm{P}=0.046 \mathrm{df} 2$, significant } \\
\hline & Paying guest & 0 & 0 & 1 & 25 & 1 & 2.1 & \\
\hline & Hosteller & 0 & 0 & 0 & 0 & 3 & 6.5 & \\
\hline \multirow[t]{2}{*}{ Previous Knowledge } & Yes & 0 & 0 & 2 & 50 & 11 & 23.91 & \multirow[t]{2}{*}{$\chi 2=1.59 \mathrm{P}=.0492 \mathrm{df} 2$, significant } \\
\hline & No & 0 & 0 & 2 & 50 & 35 & 76.08 & \\
\hline \multirow[t]{3}{*}{ Source of Information } & Magazine & 0 & 0 & 3 & 75 & 13 & 28.26 & \multirow[t]{3}{*}{$\chi 2=3.310 \mathrm{P}=0.0220 \mathrm{df} 2$, significan } \\
\hline & TV & 0 & 0 & 1 & 25 & 31 & 67.39 & \\
\hline & Friends & 0 & 0 & 0 & 0 & 2 & 4.3 & \\
\hline
\end{tabular}

\section{DISCUSSION}

The links between nutrition and health are well known, with good nutrition accepted as one of the primary determinants of optimal growth, good health and well-being. ${ }^{6}$ Accordingly, an unhealthy diet has been identified as a major risk factor for the global increase in chronic non-communicable diseases, such as coronary artery disease, cardiovascular disease, cancer, diabetes and obesity. ${ }^{7.8} \mathrm{~A}$ large proportion of these diseases can be avoided as they are either initiated or accelerated by unhealthy nutrition in addition to other etiologies. In recent years, there has been a growing worldwide concern about the dietary and nutritional needs of children. ${ }^{9}$

Our study observed that CATP was significantly effective in increasing knowledge on malnutrition and its prevention. Similar findings have also been reported in previous studies. Betageri and Tata compared the Effectiveness of structures teaching programme on knowledge regarding Integrated Child Development Services Programme among mothers of under five children. They concluded that structured teaching programme regarding ICDS programme was an effective method for providing adequate knowledge and helped mothers to enhance their knowledge and utilization of services provided under ICDS programme. Similarly, Isarannavar and Sannashivannanavar et al shows that effectiveness of structured teaching programme help to gain the knowledge score on malnutrition and the preparation of Hydrabadi and Davanagere mix recipe.

It is essential for community health nurse to develop knowledge regarding malnutrition, its management and prevention in order to avoid life threatening complications among under-five children.

\section{CONCLUSION}

Computer-assisted teaching programme (CATP) is effective in increasing knowledge of adolescent regarding malnutrition and its prevention in under-five children.

\section{REFERENCES}

1. Sarraf Z., Goldberg D., Shahbazi M., Arbuckle K., Salehi M. Nutritional status of schoolchildren in rural Iran. British Journal of Nutrition. 2005:94:390-6

2. Gorstein J., Sullivan K., Yip R., Onis M., Trowbridge F., Fajans P., Clugston G. Issues in the assessment of nutritional status using anthropometry. Bulletin of the World Health Organization. 1994;72:273-83

3. Helping India Combat Persistently High Rates of Malnutrition. http://www.worldbank.org/en/news/feature/2013/05/13/helping-indiacombat-persistently-high-rates-of-malnutrition. last accessed 17 Jun 2019.

4. Ghosh S. National Family Health Survey-3 (2007). Indian Pediatr 2007; 44:619-620

5. Institute of Medicine (US) Committee on Nutrition Services for Medicare Beneficiaries. The Role of Nutrition in Maintaining Health in the Nation's Elderly: Evaluating Coverage of Nutrition Services for the Medicare Population. Washington (DC): National Academies Press (US); 2000. 4 Undernutrition. Available from: https://www.ncbi.nlm.nih.gov/books/NBK225291/

6. Burchi F, Fanzo J, Frison E. The role of food and nutrition system approaches in tackling hidden hunger. Int J Environ Res Public Health. 2011;8:358-373.

7. Garg A, Anand T, Sharma U, et al. Prevalence of Risk Factors for Chronic Noncommunicable Diseases Using WHO Steps Approach in an Adult Population in Delhi. J Family Med Prim Care. 2014;3:112-118.

8. Al-Mawali A. Non-Communicable Diseases: Shining a Light on Cardiovascular Disease, Oman's Biggest Killer. Oman Med J. 2015;30: 227-228.

9. Gustafson DJ. Rising food costs \& global food security: key issues \& relevance for India. Indian J Med Res. 2013;138:398-410.

10. Betagiri K, Tata S. The Effectiveness of Structured Teaching Programme on Knowledge Regarding ICDS Programme among Mothers of under Five Children. IJSR. 2016;5:699-701

11. Gangadhar. S. Isarannavar et al. Effectiveness of Structured Teaching Programme on Malnutrition and the Preparation of Hydrabadi and
Davanagere Mix Recipe among Mothers of under Five Children in Selected Anganwadi centre of Hattikeri P. H. C., Ankola (U.K.), Karnataka. International Journal of Science and Healthcare Research. 2017;2:42-45 